\title{
Computer Simulations of Electron Transport through a Nanowire Quantum Dot
}

\author{
D. Łapa, A. Sowa And J. Adamowski \\ Faculty of Physics and Applied Computer Science \\ AGH University of Science and Technology \\ al. Mickiewicza 30, 30-059 Kraków, Poland
}

\begin{abstract}
Electron tunneling through a quantum dot embedded in a nanowire was studied by the transfer matrix method. A smoothness of the interfaces was taken into account using the analytical parametrization of the potential profile. We calculated the current-voltage characteristics and discussed the effect of the spacer, which separates the quantum dot from the contacts. We found that the tunneling current peak possesses the asymmetric Fano resonance shape in the absence of spacer. The results of calculations agree with the experimental data.
\end{abstract}

PACS numbers: 73.63.Nm

\section{Introduction}

Semiconductor nanowires, as quasi-one-dimensional conductors, are very promising candidates for constructing nanodevices of future electronics and quantum computing. Recently, the nanowires with single [1] and multiple [2] quantum dots (QDs) have been studied experimentally. These nanowire QDs can be used to control the resonant electron tunneling in the semiconductor nanodevices. The nanodevice made from the $\mathrm{InP} / \mathrm{InAs} / \mathrm{InP}$ double barrier heterostructure grown within the InAs nanowhisker [1] can be applied as a resonant tunneling diode (RTD). The current-voltage characteristics of the nanowire RTD exhibit a pronounced current peak with an extremely large peak-to-valley ratio (50:1) and a negative differential resistance [1].

In the present paper, we investigate the electron tunneling through the nanowire with the single QD by the transfer matrix method. Instead of the commonly used rectangular approximation of the barrier potential, we approximate it by a power-exponential potential [3], which takes into account a softness of potential barriers. The necessity of using the soft potential barriers results from the interface smoothness [4], i.e., the non-perfect sharpness of atomic planes observed at the InAs/InP interface [1], and the tensile strain field, which acts near the 
interface due to the lattice mismatch [5]. The application of the potential with the variable softness allows us to model the realistic profile of the confinement potential in the nanowire QD.

\section{Theory}

In the heterostructure [1], the potential-barrier (well) regions are formed from InP (InAs) embedded in the nanowire, which is fabricated from $n$-InAs. This nanostructure forms the quasi-one-dimensional (1D) nanowire QD. The potential profile in the nanowire QD (Fig. 1) can be modeled by the two-center power-exponential function [3]:

$$
U(z)=U_{0}\left\{\mathrm{e}^{-\left[\left(z+z_{0}\right) / R\right]^{p}}+\mathrm{e}^{-\left[\left(z-z_{0}\right) / R\right]^{p}}\right\}
$$

where coordinate $z$ is measured along the nanowire axis and $U_{0}$ is the height of potential energy barrier. Parameter $p$ determines a softness of the potential. For $p=2$ the potential is "soft" (in this case, we are dealing with two Gaussians centered at $\pm z_{0}$ ) and for $p \geq 4$ the potential can be treated as "hard" [in particular, for $p \longrightarrow \infty$ potential (1) goes over into the two rectangular potential barriers]. Range $R$ of potential (1) determines the thickness $b$ of the barrier (for the rectangular barrier $b=2 R)$.

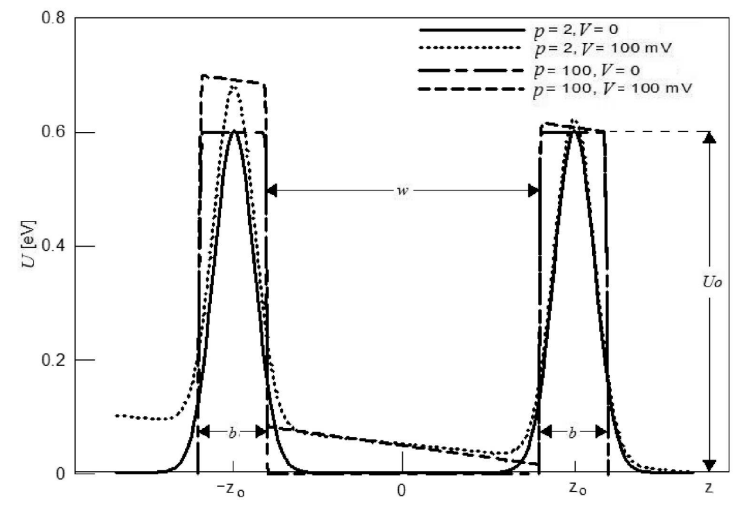

Fig. 1. Electron potential energy $U$ as a function of coordinate $z$ measured along the nanowire axis for $p=2$ and $p=100$ and for bias voltage $V=0$ and $100 \mathrm{mV}$. Parameters $b$ and $w$ determine the thicknesses of potential barrier and well, respectively, $U_{0}$ is the height of the potential barrier.

Bias voltage $V$ applied between the ohmic contacts attached to the nanowire modifies the potential shape (cf. Fig. 1), which leads to a flow of current. At low temperature, the current is mainly due to the quantum tunneling of electrons through the double-barrier region. In the $1 \mathrm{D}$ model of the nanostructure, the zero-temperature current can be expressed as follows: 


$$
I=\frac{2 e}{h} \int_{e V}^{e V+E_{\mathrm{F}}} \mathrm{d} E T(E, V),
$$

where $E_{\mathrm{F}}$ is the Fermi energy of conduction band of the degenerate $n$-InAs and $T(E, V)$ is the transmission coefficient. Throughout the present paper, we put the electrostatic potential and the energy of the conduction-band minimum of the right side of the nanodevice equal to zero. In the voltage regime near the transmission maximum, the electron transport from the right contact to the left contact is negligibly small.

\section{Results and discussion}

We have calculated the transmission coefficient as a function of energy $E$ of incident electrons and bias voltage $V$ by the transfer matrix method. In order to apply this method, the potential energy (Fig. 1) has been approximated by the piecewise constant function, for which the transfer matrix method is straightforward. We have studied the effect of spacer between the left (right) contact and the left (right) barrier. In the present calculations, we put thickness $s$ of the spacer to be either zero or maximal, i.e., $s=s_{\max }=(L-2 b-w) / 2$, where $L$ is the length of the nanowire. Due to the small thickness of InP barriers, we assume that the electron has the conduction band mass of InAs, i.e., $m_{\mathrm{e}}=0.0265 m_{\mathrm{e} 0}$, where $m_{\mathrm{e} 0}$ is the free electron rest mass. We have taken into account the smearing of each InP/InAs interface by 2 lattice spacings [1], which leads to $R=2.0625 \mathrm{~nm}$. Moreover, we fix other nanodevice parameters at their nominal values [1], i.e., $U_{0}=600 \mathrm{meV}$ and $w=15 \mathrm{~nm}$.

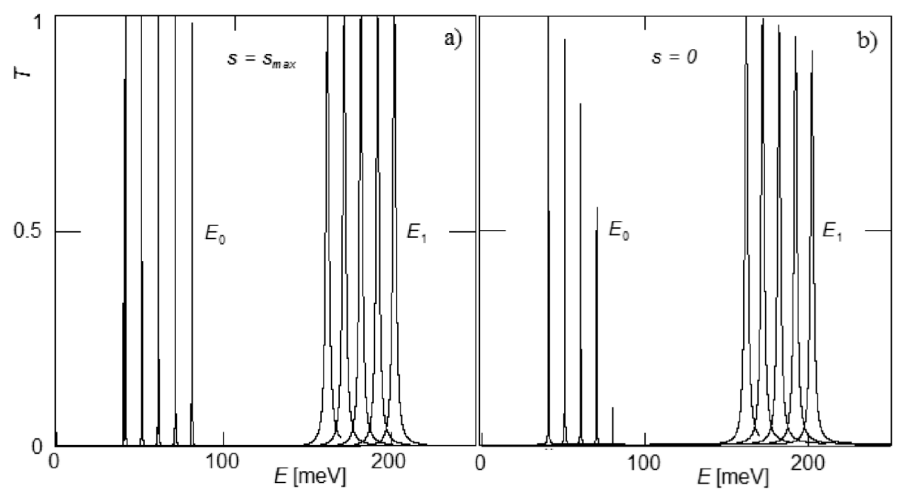

Fig. 2. Transmission coefficient $T$ as a function of incident electron energy $E$ for softness parameter $p=8$ and spacer width (a) $s=987.5 \mathrm{~nm}$, and (b) $s=0$. Each series of peaks results from the tunneling via the quasi-bound states with energies $E_{0}$ and $E_{1}$. In each series, the first left peak is plotted for bias voltage $V=0$ and the next peaks are plotted for $V=20,40,60$, and $80 \mathrm{mV}$. 


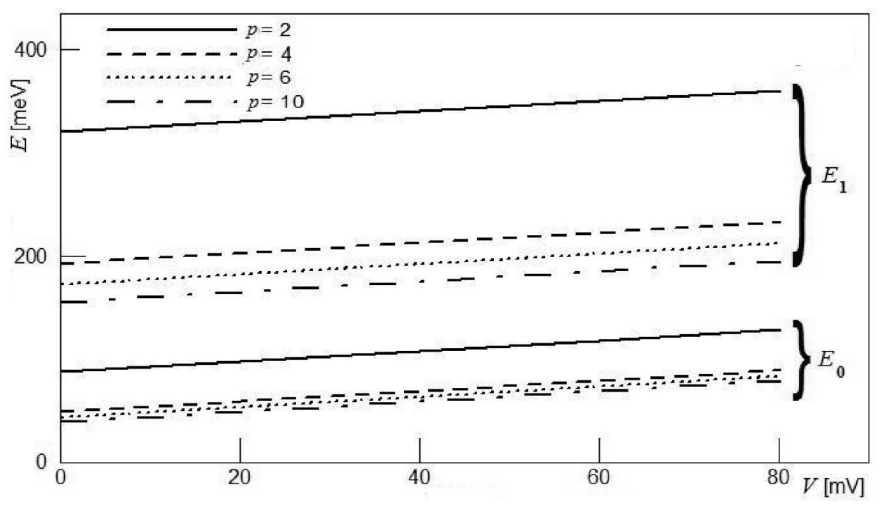

Fig. 3. Ground-state $\left(E_{0}\right)$ and excited-state $\left(E_{1}\right)$ energy levels as functions of bias voltage $V$ and softness parameter $p$.

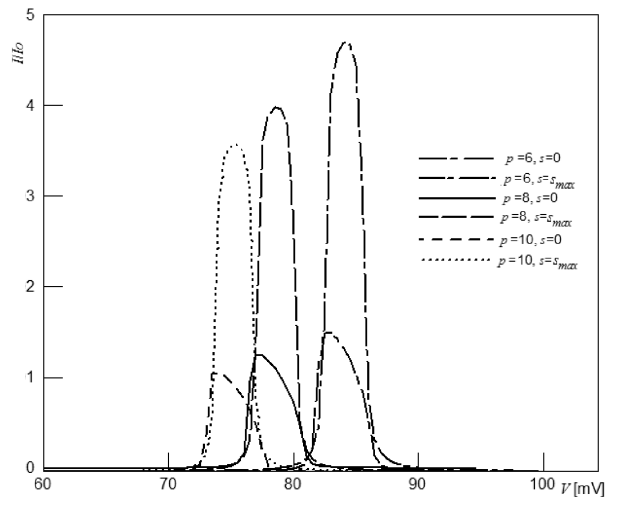

Fig. 4. Current-voltage characteristics as functions of softness parameters $p$ and spacer thickness $s$ for $E_{\mathrm{F}}=2.5 \mathrm{meV}$ and $I_{0}=7.748 \mathrm{pA}$.

The transmission coefficient calculated as a function of incident-electron energy $E$ and bias voltage $V$ is plotted in Fig. 2a (for $s=s_{\max }$ ) and Fig. 2b (for $s=0)$. The sharp transmission peaks result from a resonant tunneling of electrons through the quasi-bound states in the QD. The energy positions of these peaks very well agree with energy levels $E_{n}$ of the quasi-bound states calculated by the imaginary time-step method. Figure 3 depicts the ground-state $\left(E_{0}\right)$ and first-excited state $\left(E_{1}\right)$ energy levels as functions of bias voltage $V$. The transmission peaks (Fig. 2) shift toward higher energies if the bias voltage increases. This shift results from the corresponding increase in energy levels $E_{n}$ (cf. Fig. 3). The current-voltage characteristics calculated with the help of Eq. (2) are displayed in Fig. 4. Each current-voltage characteristic exhibits a pronounced peak if the ground-state energy $E_{0}$ belongs to the transmission window, i.e., the energy interval $\left[e V, e V+E_{\mathrm{F}}\right]$. Changing the bias voltage we change the condition of the 
resonance tunneling. The position of the current peak shifts toward the higher voltage if the potential barrier becomes more soft, which corresponds to the similar shift of energy $E_{0}$ as a function of softness parameter $p$ (cf. Fig. 3).

Figure 4 shows that the current peaks are either symmetric (for $s=s_{\max }$ ) or asymmetric (for $s=0$ ). This change of shape of the current-voltage characteristics results from the difference between the transmission peaks (cf. Fig. 2). In the case of $s=s_{\max }$, the transmission maxima are close to 1 in the resonance regime, i.e., for $V \leq 80 \mathrm{mV}$ (Fig. 2a). This leads to the symmetric shape of current peaks, which were observed in the experiment [1]. However, for $s=0$ the transmission maxima rapidly fall down to zero with the increasing voltage (Fig. 2b). In this case, the transmission energy window quickly crosses the energy level $E_{0}$ when the voltage $V$ increases. Therefore, the tunneling current is considerably smaller than in case of non-zero spacer and the current peak acquires the asymmetric Fano shape [6].

Figure 5 shows the comparison of the calculated and measured currentvoltage characteristics for the InAs/InP nanowire QD. The best agreement with the experimental data [1] has been found for $p=6.5$ and $E_{\mathrm{F}}=3.5 \mathrm{meV}$ (with all other nanodevice parameters fixed at their nominal values). The present calculations reproduce the position and half width of the current peak, but not the maximum current value and the increase in the current observed in the regime $V>100 \mathrm{mV}$. In this voltage regime, we are dealing with the phonon-assisted tunneling and thermoionic current, which were neglected in the present approach.

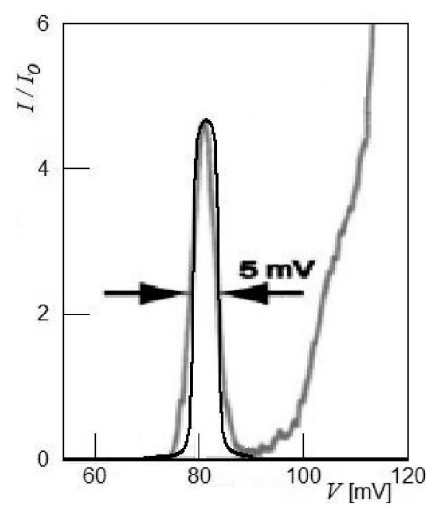

Fig. 5. Calculated (black curve) and measured (gray curve) current-voltage characteristics. The adjusted parameters are $p=6.5$ and $E_{\mathrm{F}}=3.5 \mathrm{meV}$.

In summary, we have studied the tunneling current via the QD embedded in the nanowire. Taking into account the realistic profile of the confinement potential in the framework of the $1 \mathrm{D}$ model, we have calculated the current-voltage characteristics of the nanodevice. We have found that the tunneling current peak has the asymmetric Fano shape if there is no spacer between the contacts and the 
double-barrier region, i.e., there is a strong coupling between the continuum states of electrons in contacts and the localized electron state in the QD. The present model leads to a good agreement between the calculated and measured tunneling current peaks in the nanowire QD.

\section{Acknowledgments}

This paper was partly supported by the Polish Ministry for Science and Higher Education.

\section{References}

[1] M.T. Björk, B.J. Ohlsson, T. Sass, A.I. Persson, C. Thelander, M.H. Magnusson, K. Deppert, L.R. Wallenberg, L. Samuelson, Appl. Phys. Lett. 81, 4458 (2002).

[2] A. Fuhrer, C. Fasth, L. Samuelson, Appl. Phys. Lett. 91, 052109 (2007).

[3] M. Ciurla, J. Adamowski, B. Szafran, S. Bednarek, Physica E 15, 261 (2002).

[4] V. Mlinar, A. Schliwa, D. Bimberg, F.M. Peeters, Phys. Rev. B 75, 205308 (2007).

[5] Y.-M. Niquet, D.C. Mojica, Phys. Rev. B 77, 115316 (2008).

[6] J.U. Nöckel, A.D. Stone, Phys. Rev. B 50, 17415 (1994). 